\title{
Cyclic Reversal of Magnetic Cloud Poloidal Field
}

\author{
Y. Li • J.G. Luhmann • B.J. Lynch · E.K.J. Kilpua
}

Received: 24 August 2010 / Accepted: 4 February 2011 / Published online: 4 March 2011

(C) The Author(s) 2011. This article is published with open access at Springerlink.com

\begin{abstract}
Coronal mass ejections (CMEs) carry magnetic structure from the low corona into the heliosphere. The interplanetary CMEs (ICMEs) that exhibit the topology of helical magnetic fluxropes are traditionally called magnetic clouds (MCs). MC fluxropes with axis of low (high) inclination with respect to the ecliptic plane have been referred to as bipolar (unipolar) MCs. The poloidal field of bipolar MCs has a solar cycle dependence. We report a cyclic reversal of the poloidal field of low inclination MC fluxropes during 1976 to 2009. The MC poloidal field cyclic reversal on the same time scale of the solar magnetic cycle is evident over three sunspot cycles. Approximately $48 \%$ of ICMEs are MCs, and $40 \%$ of IMCs are bipolar MCs during solar cycle 23. The speed of the bipolar MCs has essentially the same distribution as all ICMEs, which implies that they are not from any special type of CMEs in terms of the solar origin. Although CME fluxropes may undergo a number of complications during the eruption and propagation, a significant group of MCs retains sufficient similarity to the source region magnetic field to posses the same cyclic periodicity in polarity reversal. The poloidal field of bipolar MCs gives the out-of-ecliptic-plane field or $B_{z}$ component in the IMF time series. MCs with southward $B_{z}$ field are particularly effective in causing geomagnetic disturbances. During the solar minima, the $B_{z}$ field IMF sequence within MCs at the leading portion of a bipolar MC is the same with the solar global dipole
\end{abstract}

Electronic supplementary material The online version of this article

(doi:10.1007/s11207-011-9722-9) contains supplementary material, which is available to authorized users.

Y. Li (ه) · J.G. Luhmann · B.J. Lynch

Space Sciences Laboratory, University of California, Berkeley, CA, USA

e-mail: yanli@ssl.berkeley.edu

J.G. Luhmann

e-mail: jgluhman@ssl.berkeley.edu

B.J. Lynch

e-mail: blynch@ssl.berkeley.edu

E.K.J. Kilpua

Department of Physics, Division of Atmospheric Sciences and Geophysics, University of Helsinki,

Helsinki, Finland

e-mail: kilpua@ helsinki.fi 
field. Our finding shows that MCs preferentially remove the like polarity of the solar dipole field, and it supports the participation of CMEs in the solar magnetic cycle.

Keywords Coronal mass ejection $\cdot$ Magnetic cloud $\cdot$ Magnetic field $\cdot$ Solar cycle $\cdot$ Solar wind

\section{Introduction}

The magnetic structure of coronal mass ejections (CMEs) originates at the low atmosphere of the Sun, where a stressed coronal magnetic field develops at the magnetic polarity inversion lines (PILs) (see, e.g., Falconer, Moore, and Gary, 2003, 2007; Welsch and Li, 2008; Li et al., 2008, 2010; Welsch et al., 2009). The interplanetary coronal mass ejections (ICMEs) that exhibit the topology of helical magnetic fluxropes are referred to as magnetic clouds (MCs) (Burlaga et al., 1981; Burlaga, 1982, 1988; Klein and Burlaga, 1982; Gosling, 1990; Bothmer and Schwenn, 1998). MCs have been the focus of numerous studies with interests in both CMEs and the space weather (see, e.g., Zhang and Burlaga, 1988; Lepping, Burlaga, and Jones, 1990; Webb et al., 2000; Leamon, Canfield, and Pevtsov, 2002; Cremades and Bothmer, 2004; Lynch et al., 2005, 2010; Huttunen et al., 2005; Kilpua et al., 2009a, 2009b).

Evidence has been found for a dependence of the magnetic field of MCs on the phase of the solar cycle (Zhang and Burlaga, 1988; Bothmer and Schwenn, 1998; Bothmer and Rust, 1997; Mulligan, Russell, and Luhmann, 1998). When MCs pass the observer, the time sequence of the IMF often shows a large component of north - south field (or $B_{z}$ ) within the MCs, which can be northward in the leading portion and southward in the trailing portion as NS MCs, or the reverse as SN MCs. Zhang and Burlaga (1988) studied 19 MCs near the 1980 solar maximum of cycle 21, SN MCs outnumbered NS ones 13 to 6 . Bothmer and Schwenn (1998) found 34 SN MCs but 12 NS MCs in Helios spacecraft during years 1974 to 1981. Bothmer and Rust (1997) also found that SN MCs were preferentially observed (19 out of 24) during 1974 to 1981, whereas during 1982 to 1991 NS MCs were predominant (14 out of 17) in OMNI solar wind data. The MC fluxrope chirality (or the sign of helicity) is uniformly distributed regardless of the phase of the solar cycle. Bothmer and Schwenn (1998) and Bothmer and Rust (1997) attributed their findings to the magnetic field within and around disappearing solar prominences/filaments.

Mulligan, Russell, and Luhmann (1998) called NS and SN MCs "bipolar" MCs, and also they identified MCs containing only southward or northward $B_{z}$, which they named "unipolar" MCs. Bipolar MCs represent those with fluxrope axis of low inclination to the ecliptic plane, and unipolar MCs represent those with axis of high inclination to the ecliptic plane. These authors identified 35 bipolar MCs and 24 unipolar MCs at 0.7 AU in nine years of Pioneer Venus Orbiter (PVO) solar wind data from 1979 to 1988. They showed a clear solar cycle evolution of the bipolar MCs in agreement with earlier findings. In addition, they claimed that bipolar MCs preferentially occur from near the solar minimum to the solar maximum, and unipolar MCs preferentially occur after the solar maximum for solar cycle 21 . The chirality of the MCs was not ordered with the solar cycle, as found by other authors. Mulligan, Russell, and Luhmann (1998) argued for an alternative interpretation for the MC solar cycle dependence, i.e., the MC fluxrope orientation is ordered by the global solar magnetic field, where the coronal helmet streamer belt (or the source-surface neutral line) is more equatorial on the rising phase, but more tilted on the declining phase of the solar cycle. Li and Luhmann (2004) and Huttunen et al. (2005) extended the study into solar 
cycle 23 using Wind and ACE data from 1995 to 2003, finding SN MCs being preferentially observed in that period.

These findings were either in partial sunspot cycles or from data with frequent data gaps. Further evidence spanning multiple solar cycles is needed to support cyclic evolution. Now Wind and ACE spacecraft at the L1 point upstream of Earth have solar wind and IMF observations with high quality and continuity spanning over 15 years. In this study we analyze the 15 years of data from 1995 to 2009 with consistent criteria, so that our results are free from observer bias due to either different observers or change/improvement of view of the same observer, since there are no universal criteria for ICME and MC event selections (see, e.g., Gosling, 1990).

\section{Data and Definitions}

Wind and ACE solar wind (proton temperature, density and bulk speed) and IMF vector hourly averaged data during 1996 to 2009 are the major data set for this study. We analyze the data in GSE coordinates, where the $X$-axis points from the Earth to the Sun, the $Y$-axis to the east (dusk) and the $Z$-axis to the north. To identify MCs, we require

i) A low variance magnetic field with a coherent rotation of the field vector over a time interval on the order of a day.

ii) An enhanced magnetic field magnitude greater than $\approx 10 \mathrm{nT}$.

iii) A lower than average proton temperature.

These criteria are consistent with Burlaga (1991), Bothmer and Rust (1997), Bothmer and Schwenn (1998) and Mulligan, Russell, and Luhmann (1998). MCs have been described by eight types of idealized cylindrical fluxropes involving combinations of the poloidal and axial field as shown in Figure 1. These idealized fluxropes are adapted and modified after Bothmer and Schwenn (1998), Zhao and Hoeksema (1996) and Mulligan, Russell, and Luhmann (1998). In reality the orientations of CME/MC fluxropes and magnetic PILs in CME source regions vary over the range of these orientations (see, e.g., Cremades and Bothmer, 2004; Lynch et al., 2003; Yurchyshyn et al., 2007). Here the horizontal cylinder fluxropes (upper panel) represent the extreme of low inclination MCs and vertical ones (lower panel) the extreme of high inclination MCs. The fluxropes with low inclination give bipolar MCs, and the fluxropes with high inclination give unipolar MCs in the IMF north - south field time sequence. SEN (south-east-north) indicates the sequence of the field direction when a MC passes the observer at the leading edge, the fluxrope axis and the trailing edge, and similarly for SWN (south - west - north), and so on. LH stands for left-handed chirality of the fluxrope, and RH for right-handed chirality. Two classic bipolar MC events are shown in Figure 2, where, from top to bottom, we have solar wind proton temperature, proton density, bulk speed, three components of IMF and the magnitude of IMF. As indicated in the $B_{z}$ component, the left panel of Figure 2 is for a SN MC and the right panel is for a NS MC.

We identified a total of $164 \mathrm{MC}$ events in 1996 to 2009. We then follow the classification in Figure 1 for bipolar and unipolar MCs, and the sense of rotation (chirality) of the fluxrope. Under this classification, the MC magnetic field rotation is seen in the $Y-Z$ plane with visually identifiable chirality. We refer to these cases as classic MCs herein, and 99 classic MCs are identified in 1996 to 2009. We refer to the remaining 65 MCs as non-classic MCs, which are not one of the eight types of MCs in Figure 1. Specifically, they do not simply have a bipolar or unipolar $B_{z}$ field that forms apparent left-handed or right-handed helical field rotation with $B_{y}$. The magnetic field of a non-classic MC is either due to the parental 
Idealized Fluxrope

Low Inclination
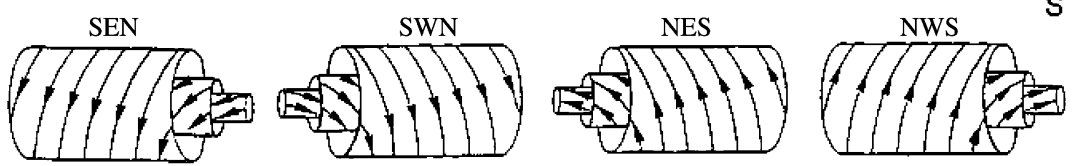

High Inclination

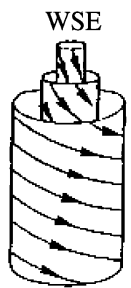

LH

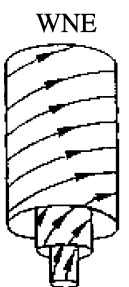

$\mathrm{RH}$

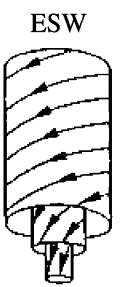

RH

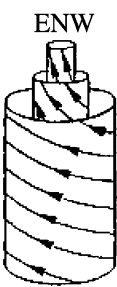

LH

Figure 1 Idealized fluxropes modified after Bothmer and Schwenn (1998), Zhao and Hoeksema (1996) and Mulligan, Russell, and Luhmann (1998). The low inclination fluxropes (left column) give bipolar MCs, and the high inclination fluxropes give unipolar MCs in the IMF time sequence. LH stands for left-handed chirality, and RH for right-handed chirality.
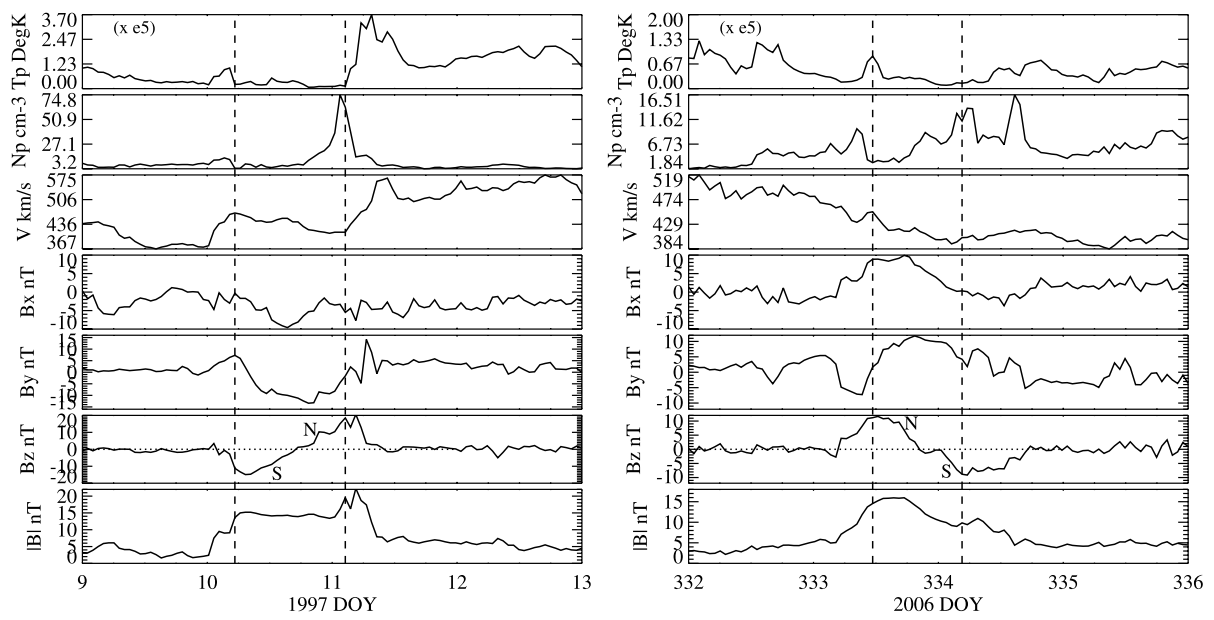

Figure 2 Time series of solar wind and IMF parameters of two bipolar classic MCs of SN (left) and NS (right) $B_{z}$ field. From top to bottom, solar wind proton temperature, proton density, bulk speed, three components of IMF and the magnitude of IMF.

CME magnetic structure being different than a cylindrical fluxrope, distortions during the propagation through the solar wind, or from other complications during the propagation. Our selection of MC events is listed in the table as an electronic attachment, where, for each year, the events are listed in the order of bipolar MCs and unipolar MCs with several MC parameters and identified MC type and chirality, and then non-classic MCs with unidentified MC type and chirality. In Table 1, we summarize the annual counts of our MCs in 1996 to 2009. We also include ICME annual counts in 1996 to 2006 by Richardson and Cane 
Table 1 Magnetic clouds and ICMEs 1996 to 2009.

\begin{tabular}{rrrrrr}
\hline Year & ICMEs $^{\mathrm{a}}$ & MCs & Classic MCs & \multicolumn{2}{c}{ Fluxrope inclination } \\
\cline { 4 - 6 } & & & & Low & High \\
\hline 1996 & 4 & 4 & 2 & 2 & 0 \\
1997 & 22 & 13 & 9 & 6 & 3 \\
1998 & 36 & 21 & 14 & 8 & 6 \\
1999 & 33 & 10 & 6 & 4 & 2 \\
2000 & 51 & 15 & 8 & 5 & 3 \\
2001 & 48 & 24 & 14 & 7 & 7 \\
2002 & 27 & 11 & 7 & 5 & 2 \\
2003 & 22 & 11 & 5 & 3 & 2 \\
2004 & 21 & 12 & 8 & 5 & 3 \\
2005 & 31 & 16 & 8 & 6 & 2 \\
2006 & 13 & 7 & 5 & 5 & 0 \\
2007 & 8 & 6 & 3 & 2 & 1 \\
2008 & 9 & 4 & 3 & 3 & 0 \\
2009 & 10 & 10 & 7 & 4 & 3 \\
& & & & 65 & 34 \\
\hline
\end{tabular}

(2010), in 2007 and 2008 by Kilpua et al. (2009b), and in 2009 by setting ICME counts equal to our MC counts. The annual counts of ICMEs identified by Richardson and Cane (2010) for 2007 to 2009 are fewer than our MC annual counts.

\section{Solar Cycle Dependence of Classic MCs}

Among the total of 99 classic MCs in 1996 to 2009 (see previous section), 65 are bipolar MCs (low inclination fluxropes), and 34 are unipolar MCs (high inclination fluxropes). In this section, we present our results of the bipolar MC polarity and MC fluxrope inclination and their evolution in three sunspot cycles by combining with previous findings.

\subsection{Poloidal Field of Low Inclination MCs}

We find 22 SN MCs and three NS MCs from the solar minimum in 1996 to maximum in 2000 (the rising phase of solar cycle 23), nine SN and six NS MCs in the next three years after the maximum in 2000 (early declining phase), and 24 NS MCs and two SN MCs from 2004 to 2009. The $B_{z}$ field component of bipolar MCs is given by the poloidal field of low inclination fluxropes. We will be using the term poloidal field and $B_{z}$ field of bipolar MCs interchangeably as best fits in the context.

Combining our current results from 1996 to 2009 and previously published results, we present, in Figure 3, the annual counts of NS (top panel) and SN (second panel) bipolar MCs. The combined time interval spans 34 years from 1976 to 2009 over three sunspot cycles or one and a half magnetic cycles (Hale cycle). The annual counts during 1979 to 1988 of PVO data are adopted from Mulligan, Russell, and Luhmann (1998), and the results during 1976 to 1978 and 1989 to 1993 of the OMNI data are adopted from Bothmer and Rust (1997). We inspected the OMNI data in 1994 and 1995 for bipolar MCs. OMNI data in the two intervals with grey bars had frequent gaps, and the MC counts are most likely incomplete. Despite 

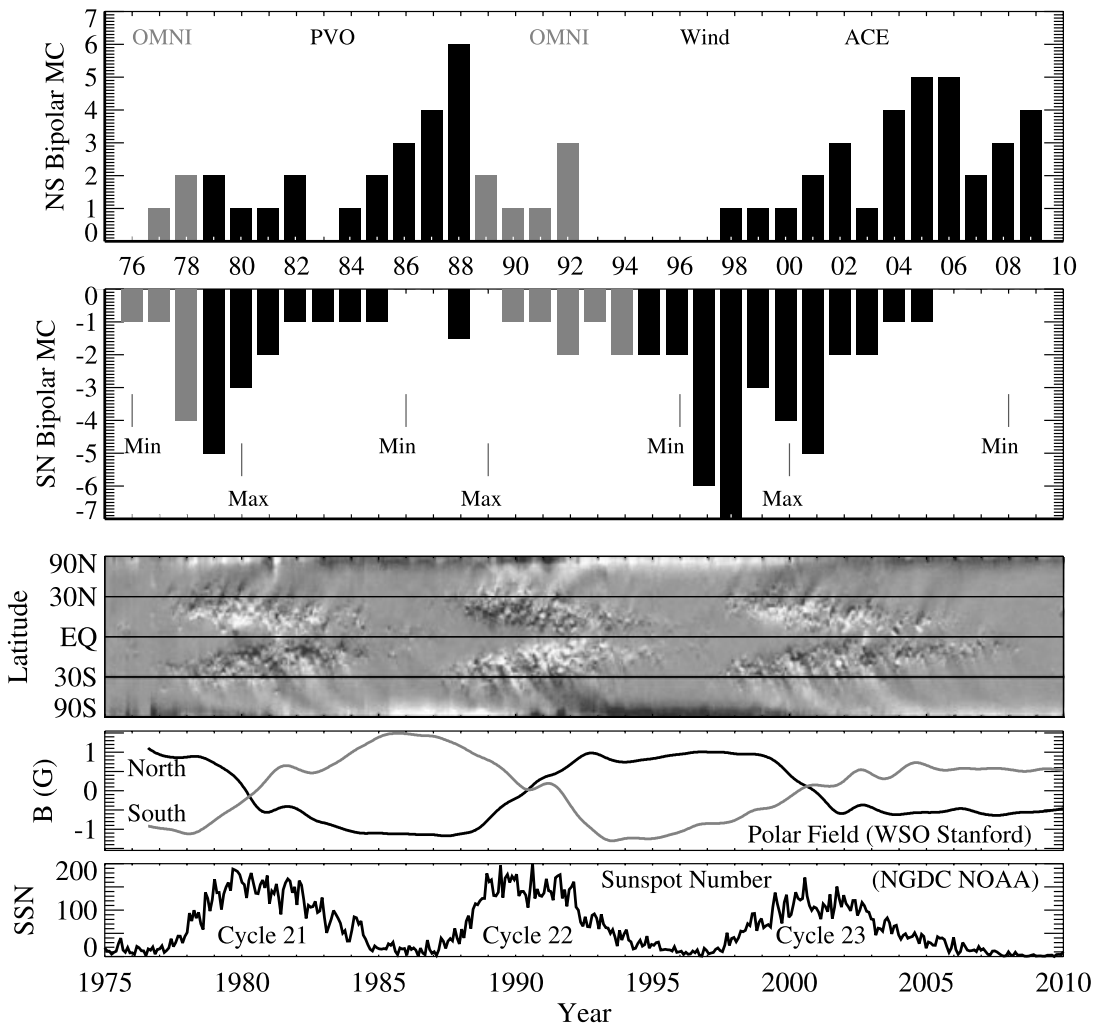

Figure 3 Two top panels: the annual counts of NS and SN bipolar MCs; Third panel: the magnetic butterfly diagram in grey scale (the original figure is by D.H. Hathaway, NASA/MSFC), where white (black) represents positive (negative) field; Forth panel: solar polar region field (between $\pm 55^{\circ}$ and the poles) observed by Wilcox Solar Observatory (WSO) at Stanford; bottom panel: sunspot number of the same three cycles 21 to 23 using data from NOAA NGDC. The poloidal field of bipolar MCs shows a cyclic reversal of the same periodicity with the solar magnetic cycle.

the data issue, the results still qualitatively support the trend. Wind and ACE data from the beginning of solar cycle 23 have the best quality with few problems and data gaps.

The third panel of Figure 3 is the magnetic butterfly diagram in grey scale, where white represents positive field and black represents negative field. The original magnetic butterfly diagram is by D.H. Hathaway, NASA/MSFC at http://solarscience.msfc.nasa.gov/images/ magbfly.jpg. The diagram includes data for the most recent three sunspot cycles and these are all the existing full disk magnetic field observations. It shows alternating polarity in the polar regions, and we see that the magnetic active regions begin to emerge at mid latitude at solar minimum and progress to lower latitude as the solar cycle proceeds. These patterns repeat themselves for the next sunspot cycle with reversed polarity which returns to the same polarity after two sunspot cycles or one solar magnetic cycle (22 years on average). The fourth panel gives the line plots of the solar polar region field (between $\pm 55^{\circ}$ and the poles) observed by the Wilcox Solar Observatory (WSO) at Stanford, and the bottom panel is the sunspot number of the same three cycles 21 to 23 using data from NOAA NGDC. These show the solar cycle manifestations of the magnetic field and intensity field at different latitudes on the photosphere. The polar region magnetic field cycle is at a reversed phase 


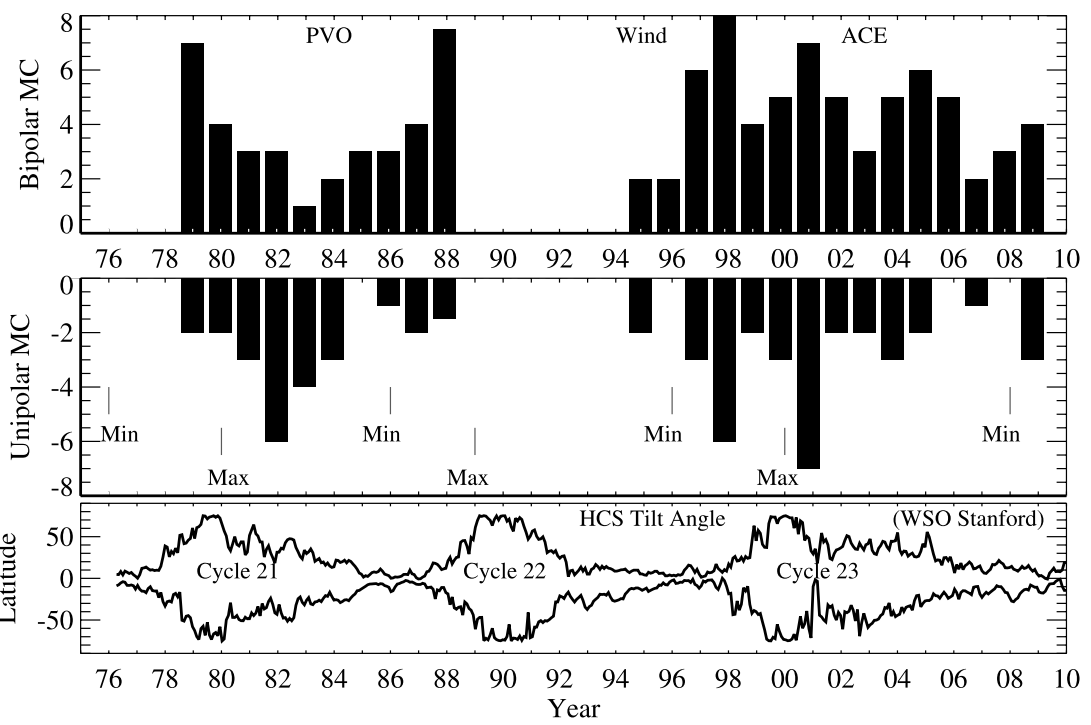

Figure 4 Top and center panels: the annual counts of bipolar and unipolar MCs from 1979 to 2009. Bottom panel: the heliospheric current sheet (HCS) tilt angle obtained by WSO of Stanford based on the potential-field source-surface (PFSS) model.

from the Hale cycle, i.e., strongest at sunspot minima. MCs appear to have the maximum occurrence on the rise phase of a sunspot cycle.

A cyclic reversal of the poloidal field of bipolar MCs is evident over the most recent three sunspot cycles. The direction of the poloidal field in the leading portion of majority bipolar MCs agrees and reverses with the global poloidal field of the Sun through most of the solar cycle, except in the few years after the solar maximum. The poloidal field polarity of MCs is mixed during the few years after the solar maximum when the solar polar field reversal takes place.

\subsection{Inclination of MC Fluxrope}

Low inclination fluxropes give bipolar MCs and high inclination fluxropes give unipolar MCs as described earlier. We use the annual counts of bipolar and the annual counts of unipolar MCs to investigate the fluxrope inclination variation with time as in Mulligan, Russell, and Luhmann (1998). We find that the MC fluxrope inclination has no solar cycle dependence in our results for cycle 23. Figure 4, top and center panel, combines our annual counts of bipolar and unipolar MCs in 1995 to 2009 with the results for 1979 to 1988 of Mulligan, Russell, and Luhmann (1998). Figure 4, bottom panel, shows the heliospheric current sheet (HCS) tilt angle obtained by WSO Stanford based on the potential-field sourcesurface (PFSS) model. The curves measure the maximum excursion of the HCS from the solar equator for each Carrington rotation (CR) at the northern (upper curve) and southern (lower curve) hemispheres. The result from PVO data taken at $0.7 \mathrm{AU}$ showed that low inclination fluxropes (bipolar MCs) preferentially occurred on the rising phase of cycle 21, and high inclination fluxropes (unipolar MCs) preferentially occurred on the declining phase. The result led to the interpretation that MC fluxrope orientation is controlled by the inclination of the coronal helmet streamer belt. The MC fluxrope inclination measured in the same 
manner in cycle 23 at $1 \mathrm{AU}$ does not show the same trend or any trend, and consequently does not support the interpretation. In the next section, we show further analysis of the MC parameters to gain a better view of the possible origin of the $\mathrm{MC}$ field and the long term trend.

\section{Further Analysis of MCs in Solar Cycle 23}

We have shown the solar cycle dependence of the classic MCs in the previous section. As described earlier, some previous studies attributed the polarity variation over the solar cycle to the erupting prominence/filament field, but those studies did not consider the inclination of the MC fluxrope. The MC polarity and inclination solar cycle dependence in solar cycle 21 can both be explained, if CMEs erupt part of the coronal streamer arcades which are subsequently observed as MCs. But the solar cycle trend of the MC fluxrope inclination does not hold for cycle 23, while the polarity solar cycle trend of low inclination MC fluxropes does clearly persist. The following questions are again raised. What is (are) the origin(s) of the magnetic structure of MCs, and how do we interpret the solar cycle dependence? In this section, we present a further analysis of the MC parameters of cycle 23 in order to further understand these questions.

As we know, MCs represent those ICMEs with organized and enhanced magnetic field. First we investigate what fraction of ICMEs gives the group of MCs in our study. In Figure 5, top panel, we plot the annual counts of classic MCs, all MCs and ICMEs with time. The solid line represents annual counts of the classic MCs. The occurrence rate of classic MCs neither shows a simple solar cycle variation, nor correlates with the sunspot cycle. The dashed line represents the annual counts of all MCs, which has a similar temporal trend with classic MCs. The dotted line represents the annual counts of ICMEs combining results

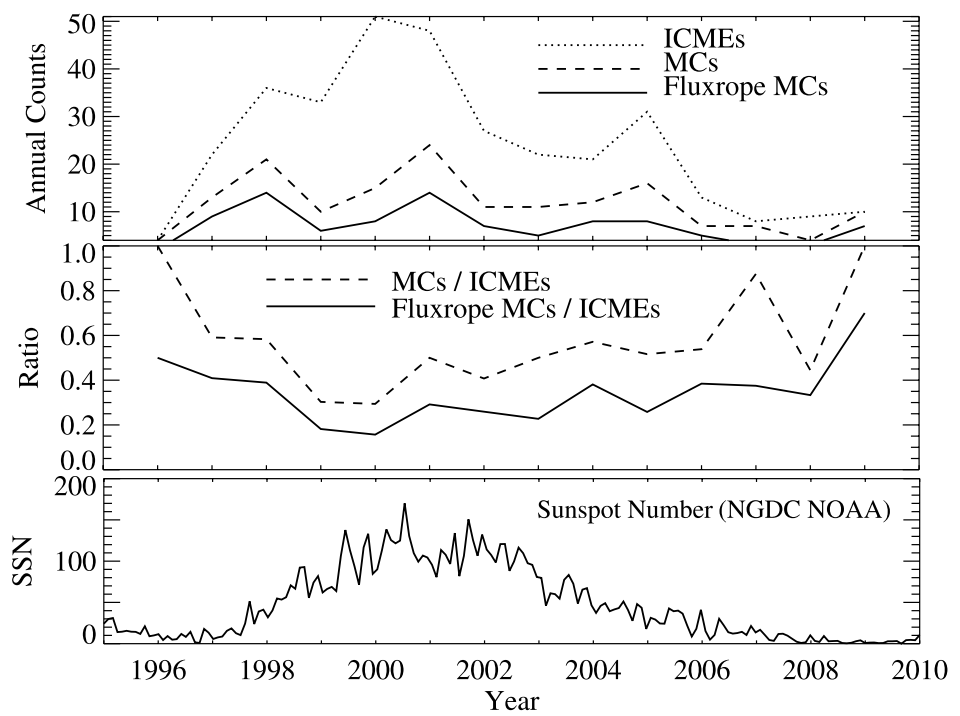

Figure 5 Top panel: the annual counts of classic MCs (solid line), MCs (dashed line) and ICMEs (dotted line); center panel: annual ratio between classic MCs and ICMEs (solid line) and between MCs and ICMEs (dashed line). Bottom panel: monthly sunspot numbers of cycle 23. 

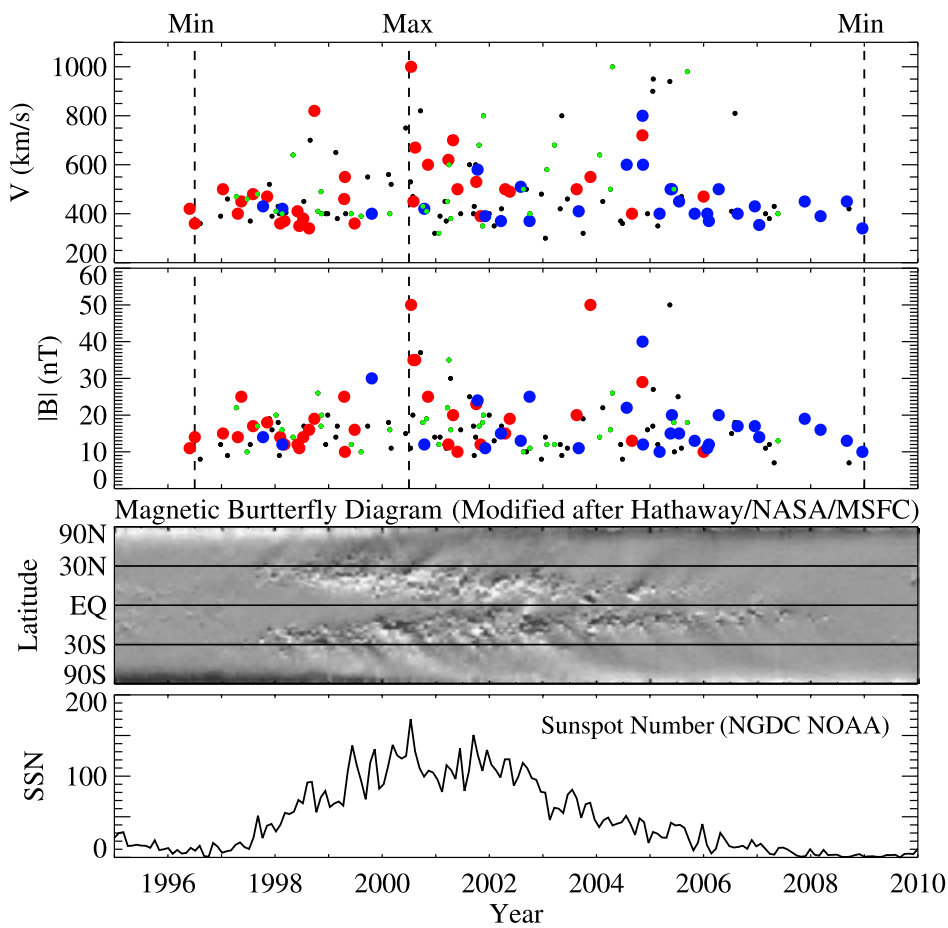

Figure 6 Top panel: temporal distribution of MC Speed. Second panel: temporal distribution of peak magnitude of magnetic field within MCs. Red filled circle: SN bipolar MCs; Blue filled circle: NS bipolar MCs; Green dots: unipolar MCs, Black dots: non-classic MCs. Third panel: magnetic butterfly diagram of cycle 23 . Bottom panel: sunspot numbers of cycle 23 .

in Richardson and Cane (2010), Kilpua et al. (2009b), and our own total MC counts in 2009 (see Section 2 for descriptions). Unlike MCs, the annual counts of ICMEs at 1 AU have a positive correlation with the sunspot cycle (bottom panel); see also Richardson and Cane (2010). In Figure 5, center panel, the solid line gives the ratio between the annual counts of the classic MCs and ICMEs, and the dashed line gives the ratio between the annual counts of all MCs and ICMEs. The ratio is higher near solar minimum, meaning a greater portion of ICMEs is of the fluxrope type, which is in agreement with findings in Cane and Richardson (2003) and Richardson and Cane (2010). Over 1996 to 2008 (solar cycle 23), we have 93 classic MCs, $155 \mathrm{MCs}$ and 325 ICMEs. The ratio between the total counts of classic MCs and ICMEs is $29 \%$, and the ratio between the total counts of MCs and ICMEs is $48 \%$. Event counts in 2009 are preliminary and are not included when calculating these ratios above. The often cited ratio that one third of ICMEs are MCs was from ISEE data in partial sunspot cycle 21 (Gosling, 1990).

In order to understand the origin of the magnetic structure of ICMEs, we investigate in further detail the magnetic field of different group of events, along with the speed of the ICMEs, because the speed of ICMEs is closely associated with different types of eruptive source regions at the low corona. In Figure 6 we present the temporal distribution of the speed of MCs (top panel), and the peak magnitude of magnetic field within the MCs (second panel). The red filled circles represent SN MCs, blue filled circles for NS MCs, green dots for unipolar MCs, and black dots for non-classic MCs. The third and bottom panels are the magnetic butterfly diagram and sunspot numbers of cycle 23 (also shown in Figure 5, 
bottom panel), respectively, co-aligned for easy reference. The speed of all MCs shows a relatively simple temporal variation. A few years before and after the solar minima around late 1996 and 2008, the speed of the MCs is below $500 \mathrm{~km} \mathrm{~s}^{-1}$. During the rest of the solar cycle, the speed of the MCs spreads well across the whole speed range. The maximum speed of all MCs is about $1000 \mathrm{~km} \mathrm{~s}^{-1}$. One of the fastest MCs occurred at solar maximum as a low inclination SN MC, and a group of the five fastest MCs occurred in 2004 to 2005 as either high inclination MCs or non-classic MCs. The peak magnetic field magnitude of 155 out of the total $164 \mathrm{MCs}$ (or 94.5\%) is below $30 \mathrm{nT}$, and only nine MCs (or 5.5\%) had a value between 30 and $50 \mathrm{nT}$. The maximum value of peak field strength of all MCs in solar cycle 23 is approximately $50 \mathrm{nT}$, the first of which was at the solar maximum in 2000 as a low inclination SN MC, the second was in 2003 as also a SN MC and the third was in 2005 as a non-classic MC.

The speed of low inclination MCs shows a more structured temporal distribution differing from that of all MCs as a whole. Both the speed and field magnitude of low inclination MCs have two peaks in 2000 and 2005. Throughout the rising phase 1996 to 2000, almost all bipolar MCs are SN ones (red), and most MCs have slow to moderate speed and field magnitude ranging 10 to $25 \mathrm{nT}$. A group of fast SN MCs with speed $\gtrsim 600 \mathrm{~km} \mathrm{~s}^{-1}$ appeared at and just after the solar maximum in late 2000, pointing to the CME origin being large sunspot regions with strong magnetic field. The MC poloidal field remains of the same polarity as before the maximum. MCs with reversed polarity (blue) begin to occur in significant numbers at slow to moderate speed about a year after the polar field reversal has taken place around the solar maximum, specifically, in 2000 at the north pole and 2001 at the south pole. MCs with both polarities coexisted in comparable numbers for a few years before the NS MCs prevailed. The poloidal field reversal of slower MCs can be explained by the solar dipole field reversal, but a few very fast MCs also show a reversed poloidal field on the declining phase, while the sunspot field morphology should be the same as at the solar maximum. Sunspot fields begin to reverse at the solar minima, and low latitude old cycle sunspots and high latitude new cycle sunspots coexist with opposite leading and following field, but the MCs in the ecliptic plane do not show mixed polarities around solar minima when bipolar MCs comprise almost all ICMEs.

We also construct the histograms of the speed of different groups of MCs and make a comparison with the speed distribution of the general ICMEs. The histograms in Figure 7 have a bin size of $50 \mathrm{~km} \mathrm{~s}^{-1}$ and are normalized to one at the peaks. The black line in each panel represents the speed distribution of a group of MC and the grey line is for the distribution of all 325 ICMEs in 1996 to 2008. The speed distribution of the 92 classic MCs is shown in the top left panel, the 65 non-classic MCs in the top right panel, the 61 bipolar (low inclination) MCs in the bottom left panel, and the 31 unipolar (high inclination) MCs in the bottom right panel. While the classic and non-classic MC speed distributions are not identical, both of them peak around $400 \mathrm{~km} \mathrm{~s}^{-1}$ and do not show any significant difference from the speed distribution of the total ICME population. The bipolar MCs also have a similar speed distribution as that of the ICMEs. The unipolar MCs show some difference in speed distribution, which may in part be due to the small number of events. The speed distributions of both bipolar and unipolar MCs peak around $400 \mathrm{~km} \mathrm{~s}^{-1}$, and they have a spread over the entire speed range.

Fast CMEs are usually concurrent with flares from sunspot regions with strong magnetic field. Slow CMEs often either originate from decayed magnetic regions with weaker field and quiescent filaments, or have no clear source regions. Based on their speed distribution, each of the subgroups of MCs can be from all types of solar origins. Of particular interest is the group of low inclination MCs, the poloidal magnetic field of which shows a persistent 

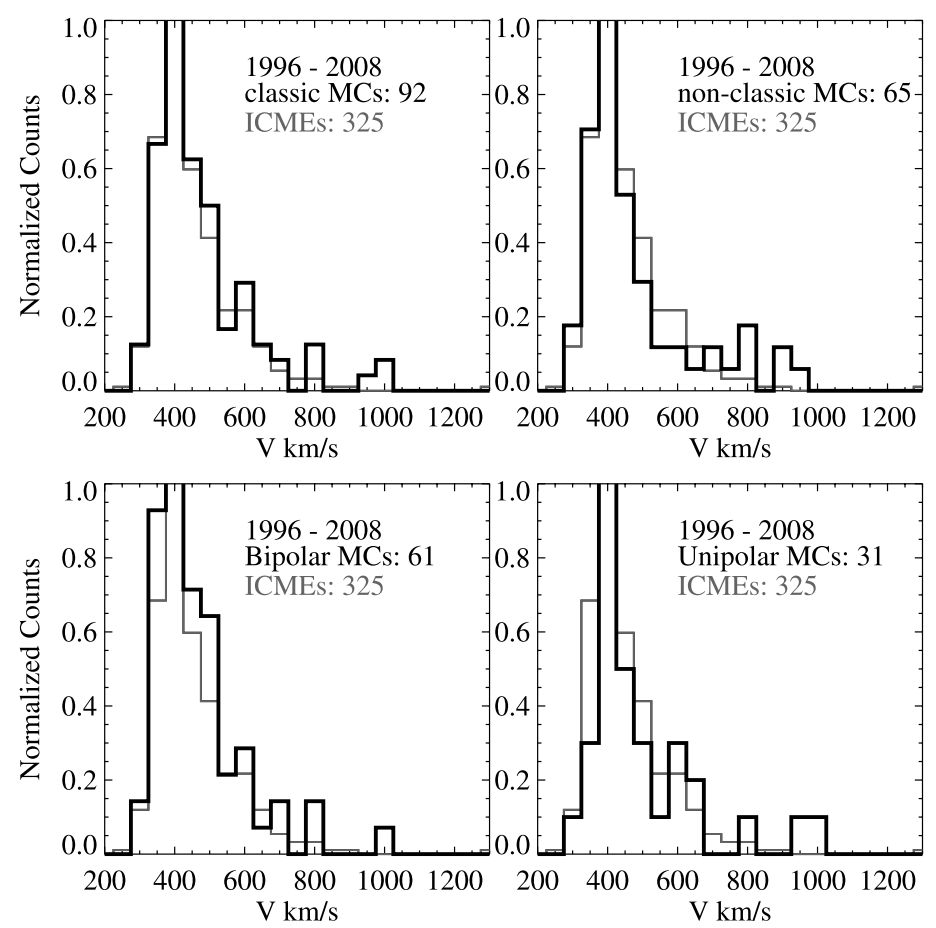

Figure 7 Top left: speed distribution of classic MCs (black) and ICMEs (grey); top right: speed distribution of non-classic MCs (black) and ICMEs (grey); bottom left: speed distribution of bipolar MCs (black) and ICMEs (grey); bottom right: speed distribution of unipolar MCs (black) and ICMEs (grey).

cyclic reversal on the solar cycle time scale. The 61 bipolar MCs, which account for $66 \%$ of classic MCs, $37 \%$ of all MCs, or 19\% of ICMEs, are not distinguished from the general population of ICMEs or MCs in kinematics, and therefore also are not from any special type of solar origin.

The sign of helicity (or chirality) of the classic MCs has no solar cycle dependence (see attachment table), which simply confirms previous findings. The randomly distributed chirality of MC fluxropes has been explained as follows: the sources of MCs may be from the southern and northern hemispheres of the Sun (Rust, 2004; Pevtsov, 2002; Bothmer and Schwenn, 1998; Bothmer and Rust, 1997). We will not further elaborate on this issue but refer the readers to the discussions in these previous studies.

\section{Discussions}

We have shown that the poloidal field of the low inclination MC fluxropes has a cyclic reversal similar to the solar dipole field and the trend extends to three sunspot cycles. We have also found that this group of MCs is not from any particular solar source regions by examining the speed distribution of the MCs. A true understanding of the association between the magnetic structure of MCs and their source regions can only be achieved when we fully understand the formation and dynamics of CMEs during the initiation and propagation. The difficulties of the coronal magnetic field observations and the sparseness of interplanetary 
field measurements need to be overcome. We have, however, a wealth of knowledge of the magnetic line-of-sight field at the photospheric level, and some understanding of CME formation and dynamics. In the next two subsections, we discuss these issues by summarizing the magnetic characteristics of possible CME source regions and previous studies that attempted to relate the source magnetic structure to the MC in situ magnetic field.

\subsection{CME Breeding Grounds}

The magnetic fluxropes that escape the Sun with some flares and prominence eruptions become CMEs and are observed as MCs in the solar wind. Prominences/filaments form at magnetic polarity inversion lines (PIL) of the photospheric radial field (Leroy, Bommier, and Sahal-Brechot, 1983; Tang, 1987; Martin, 1998; Li and Luhmann, 2006; Mackay, Gaizauskas, and Yeates, 2008). Solar flares likewise occur at PILs (see, e.g., Schrijver, 2007; Welsch et al., 2009; Li et al., 2010). By means of schematic diagrams in Figure 8, we summarize simplified distributions of PILs on the Sun at the solar minimum, rising phase, maximum and declining phase. Red (blue) PILs have a southward (northward) overlying arcade field. The solar dipole polarity in the diagrams is consistent with cycle 23 , and it should apply to all odd numbered sunspot cycles, but needs to be reversed for even num-

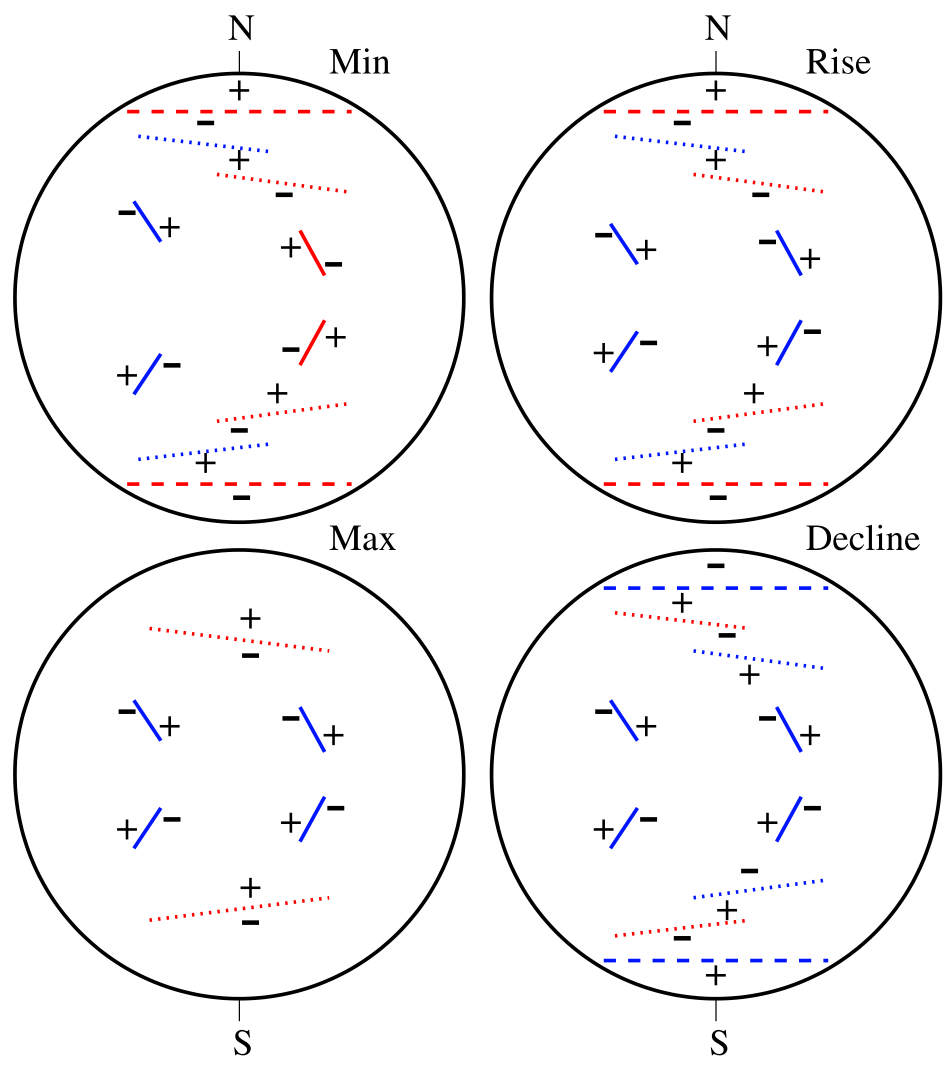

Figure 8 Schematic summary of magnetic polarity inversion lines (PILs) through sunspot cycle 23. Red: PILs with southward overlying arcades; blue: PILs with northward overlying arcades 
bered cycles. As shown, the magnetic field arcades over PILs can have both northward and southward polarities at any time through the solar cycle.

The low latitude PILs (solid lines) are in active regions (AR) within stronger fields where intense flares occur. We refer to these PILs as AR PILs for convenience. As described by Joy's Law, sunspot regions tend to be tilted with the leading spot closer to the equator than the following spot. For a short period at the solar minimum, old cycle and new cycle sunspots coexist. The old cycle sunspot regions (red solid lines) emerge near the solar equator with the leading polarity opposite from the polar region field of the same hemisphere. The new cycle sunspot regions (blue solid lines) emerge at mid latitudes with the leading polarity like the polar field of the same hemisphere. The old cycle sunspots then cease to appear. The new cycle sunspots emerge at progressively lower latitudes and more frequently. After the solar minimum, majority bipolar sunspot regions follow the same polarity pattern. There are also regions with more complex field distribution and PILs. Large sunspot active regions are often more active in generating large flares and fast CMEs. The low latitude AR field is in fact more complex and temporally variable than high latitude filament regions, and there are also PILs forming between ARs, although they are not reflected in the simplified diagrams.

The PILs at high latitudes are among decayed field, where quiescent filaments form, including polar crown filaments over the highest latitude PILs (dashed lines). We refer to these PILs as filament PILs for convenience. During the solar minimum and rising phase, polar crown filament PILs (red dashed line) have southward field arcades. Other quiescent filament PILs (dotted lines) can have field arcades of either polarity. The polar field weakens and disappears during the polar field reversal at the solar maximum, and the polar crown filament PILs also disappear, along with the adjacent filament PILs. At solar maximum there appear to be less high latitude filament PILs, the remaining filament PILs are at lower latitudes and have southward arcade. The polar field re-establishes a few years after the solar maximum with reversed polarity. At the declining phase, the polar crown filament PILs (blue dashed line) have northward overlying arcades. Filament PILs show the general trend in cycle 23 that majority PILs with southward overlying arcade (red) are at the minimum and on the rising phase, and majority PILs with northward arcades (blue) are on the declining phase.

CME source regions can have bipolar or multipolar coronal field topology. Bipolar topology means that the field forming the CME fluxrope and the overlying field are essentially a single flux system with no separatrix and null point. Multipolar topology does have a separatrix and a null point in the CME source region flux systems. Li and Luhmann (2006) found both bipolar and multipolar topology at eruptive quiescent filaments regions. The occurrence of bipolar topology cases has no solar cycle dependence, but multipolar topology cases primarily occur on the declining phase. This may contribute to, or at least appear to be consistent with, the co-existence of MCs of both polarities in the declining phase.

The direct imprint of the solar magnetic field polarity does have a solar cycle trend that is consistent with the poloidal field of MCs in the ecliptic (see Figure 3). CMEs, however, are dynamic, and they involve processes that can alter and distort the magnetic structure at their origins (see discussions in the next subsection).

\subsection{Complications of CME Fluxropes}

CME studies by means of observational and theoretical methods found that CME fluxropes undergo a number of complications in the process of their eruption and propagation.

Lynch et al. (2009) performed 3D MHD simulations of two CME eruptions. In the simulations, the two CME fluxropes only differ by having opposite signs of helicity, and it is 
found that the axis of the CME fluxrope with right-handed (left-handed) chirality rotates clockwise (counter-clockwise) during the eruption in the corona from 2 to $4 R_{\odot}$ for about $50^{\circ}$. The sense of helicity in filaments and active regions in the northern (southern) hemisphere of the Sun is preferentially left-handed (right-handed) (Pevtsov, 2002). If the rotation described in Lynch et al. (2009) applies, most CMEs from the north (south) would rotate $\mathrm{CCW}(\mathrm{CW})$. Depending on the initial PIL inclination, this effect can make the MC fluxrope inclination either lower or higher with respect to the ecliptic. Also the relationship between the source region magnetic field parameters and the degree of rotation has not been investigated. Our speculation is that it may not be simple, but may depend on both the core region and overlying coronal magnetic field. In an MHD simulation, Gibson and Fan (2008) found that the CME magnetic structure may differ significantly from the pre-eruption fluxrope due to alteration in the process of the eruption, specifically, the fluxrope undergoes writhe and both internal and external reconnections, and results in an MC structure that resembles a spheromak. While the most elaborated and applied model for MCs is the constant $\alpha$ cylindrical force-free fluxrope (Burlaga, 1988), other models such as torus (Ivanov et al., 1989) and spheromak (Vandas, Fischer, and Geranios, 1991) have also been proposed and fitted to some cases. A torus model produces a local magnetic field similar to a cylindrical fluxrope.

After comparing magnetic flux and helicity in active regions and in MCs, Leamon et al. (2004) concluded that MCs from active regions are formed by magnetic reconnection between these regions and their large scale surroundings, rather than simple eruptions of preexisting structures in the corona or chromosphere. Qiu et al. (2007) found that the magnetic flux swept up by flare ribbons agrees with the poloidal flux in MC fluxropes and the magnetic flux in CME dimming regions agrees with the toroidal flux in MC fluxropes. The authors concluded that the MC fluxrope is formed during the eruption by reconnections other than a simple ejection of a pre-existing fluxrope. Thernisien, Howard, and Vourlidas (2006) found that in most cases CME fluxropes do not expand in a self-similar manner. Yurchyshyn et al. (2007) found that 64\% CME-MC fluxrope orientation showed agreement within $45^{\circ}$. Yurchyshyn, Abramenko, and Tripathi (2009) reported that 70\% CME fluxropes in SOHO LASCO white light images rotate about $10^{\circ}$ from EUV post-flare arcades, and for some events rotation of about $30^{\circ}$ to $50^{\circ}$. They noted that CME fluxropes have a slight tendency to line up with the coronal streamer belt. In addition, CMEs interact with the solar wind and IMF during the propagation through the heliosphere, when the CME fluxrope or structure can also be distorted (see, e.g., Odstrcil, Pizzo, and Arge, 2004; Owens, 2006).

The above is by no means an inclusive review of the formidable amount of studies concerning CME and MC magnetic structure, but it is sufficient to illustrate the complications. However, quite surprisingly, the solar poloidal field polarity is retained by part of the CMEs and the polarity trend persists through decades, and is convincing enough to be expected in the future.

\section{Conclusions}

The magnetic field structure of MCs stems from the magnetic field near the solar surface. Although CME fluxropes may experience a number of complications during the eruption and propagation as described above, the fluxrope field of a significant group of CMEs reflects the magnetic field of their source regions at the low corona. The poloidal field of the low inclination MCs exhibits polarity reversal following the 22-year Hale cycle. These MCs are from the general population of CMEs in terms of their origins, i.e., their origins consist of slow CMEs usually from quiescent filament eruption and streamer blow out events, as well as fast CMEs mostly from intense flares. 
It may also be important from the space weather point of view to note the following. During the few years before solar minima, essentially all MCs have low inclination and the leading field polarity is the same as the solar dipole field. This is also true for the few years after minima, which adds up to a significant fraction of the sunspot cycle. Therefore, for several years around a solar minimum, there is a predictable $\mathrm{MC} B_{z}$ field polarity temporal sequence in the ecliptic plane, i.e., the leading field polarity encountered by an observer is always the same as the solar dipolar field around solar minima.

Finally, it is believed that CME plays a part in the solar magnetic field cyclic reversal by removing the old cycle field and helicity (Low, 2001; Gopalswamy et al., 2003). The solar cycle dependence of the low inclination MC poloidal field reinforced in three sunspot cycles shows evidence that CMEs preferentially remove the like polarity of the solar dipole field and support CME participation in the solar magnetic cycle.

Acknowledgements $\quad \mathrm{Y} . \mathrm{Li}$ and BJL acknowledge the support of NASA awards SRT NNG06GE51G and GI NNX08AJ04G. Y. Li and JGL are partially supported by NSF CISM ATM-0120950. Major data sources are ACE, Wind, OMNI, WSO Stanford polar magnetic field and HCS tilt angle and NOAA NGDC SSN. The authors thank George Fisher for valuable discussions.

Open Access This article is distributed under the terms of the Creative Commons Attribution Noncommercial License which permits any noncommercial use, distribution, and reproduction in any medium, provided the original author(s) and source are credited.

\section{References}

Bothmer, V., Rust, D.M.: 1997, In: Crooker, N., Joselyn, J.A., Feynman, J. (eds.) Coronal Mass Ejections, AGU Geophysical Monograph 99, 137.

Bothmer, V., Schwenn, R.: 1998, Ann. Geophys. 16, 1.

Burlaga, L.F.: 1988, J. Geophys. Res. 93, 7217.

Burlaga, L.F.: 1991, In: Schwenn, R., Marsch, E. (eds.) Physics of the Inner Heliosphere II. Particles, Waves and Turbulence, Springer, Berlin, 152.

Burlaga, L., Sittler, E., Mariani, F., Schwenn, R.: 1981, Solar Phys. 86, 6673.

Burlaga, L.F., Klein, L., Sheeley, N.R., Michels, D.J. Jr., Howard, R.A., Koomen, M.J., Schwenn, R., Rosenbauer, H.: 1982, Geophys. Res. Lett. 9, 1317.

Cane, H.V., Richardson, I.G.: 2003, J. Geophys. Res. (Space Phys.) 108(A4), SSH 6-1, CiteID 1156, doi:10.1029/2002JA009817.

Cremades, H., Bothmer, V.: 2004, Astron. Astrophys. 422, 307.

Falconer, D.A., Moore, R.L., Gary, G.A.: 2003, J. Geophys. Res. 108(A10), SSH11-1.

Falconer, D.A., Moore, R.L., Gary, G.A.: 2007, J. Atmos. Solar-Terr. Phys. 69, 86.

Gibson, S.E., Fan, Y.: 2008, J. Geophys. Res. 113, A09103.

Gopalswamy, N., Lara, A., Yashiro, S., Howard, R.A.: 2003, Astrophys. J. 598(1), L63 - L66.

Gosling, J.T.: 1990, In: Russell, C.T., Priest, E.R., Lee, L.C. (eds.) Physics of Magnetic Fluxropes, AGU Geophysical Monograph 58, 343.

Huttunen, K.E.J., Schwenn, R., Bothmer, V., Koskinen, H.E.J.: 2005, Astron. Astrophys. $23,625$.

Ivanov, K.G., Harshiladze, A.F., Eroshenko, E.G., Stiazhkin, V.A.: 1989, Solar Phys. 120, 407.

Kilpua, E.K.J., Liewer, P.C., Farrugia, C., Luhmann, J.G., Möstl, C., Li, Y., et al.: 2009a, Solar Phys. 254, 325.

Kilpua, E.K.J., Pomoell, J., Vourlidas, A., Vainio, R., Luhmann, J., Li, Y., Schroeder, P., Galvin, A.B., Simunac, K.: 2009b, Ann. Geophys. 27, 4491.

Klein, L.W., Burlaga, L.F.: 1982, J. Geophys. Res. 87, 613.

Leamon, R.J., Canfield, R.C., Pevtsov, A.A.: 2002, J. Geophys. Res. 107(A9), SSH 1-1.

Leamon, R.J., Canfield, R.C., Jones, S.L., Lambkin, K., Lundberg, B.J., Pevtsov, A.A.: 2004, J. Geophys. Res. 109, A05106.

Lepping, R.P., Burlaga, L.F., Jones, J.A.: 1990, J. Geophys. Res. 107, 11957.

Leroy, J.L., Bommier, V., Sahal-Brechot, S.: 1983, Solar Phys. 83, 135.

Li, Y., Luhmann, J.G.: 2004, J. Atmos. Solar-Terr. Phys. 66, 323.

Li, Y., Luhmann, J.G.: 2006, Astrophys. J. 648, 732. 
Li, Y., Lynch, B.J., Stenborg, G., Luhmann, J.G., Huttunen, K.E.J., Welsch, B.T., Liewer, P.C., Vourlidas, A.: 2008, Astrophys. J. Lett. 681, 37.

Li, Y., Lynch, B.J., Welsch, B.T., Stenborg, G.A., Luhmann, J.G., Fisher, G.H., Liu, Y., Nightingale, R.W.: 2010, Solar Phys. 254, 149.

Low, B.C.: 2001, J. Geophys. Res. 106, 25141.

Lynch, B.J., Zurbuchen, T.H., Fisk, L.A., Antiochos, S.K.: 2003, J. Geophys. Res. 108(A6), SSH6-1.

Lynch, B.J., Gruesbeck, J.R., Zurbuchen, T.H., Antiochos, S.K.: 2005, J. Geophys. Res. 110, A08107.

Lynch, B.J., Antiochos, S.K., Li, Y., Luhmann, J.G., DeVore, C.R.: 2009, Astrophys. J. 697, 1918.

Lynch, B.J., Li, Y., Thernisien, A.F.R., Robbrecht, E., Fisher, G.H., Luhmann, J.G., Vourlidas, A.: 2010, J. Geophys. Res. 115, A07106.

Mackay, D.H., Gaizauskas, V., Yeates, A.R.: 2008, Solar Phys. 248, 51.

Martin, S.F.: 1998, Solar Phys. 182, 107.

Mulligan, T., Russell, C.T., Luhmann, J.G.: 1998, J. Geophys. Res. 25, 2959.

Odstrcil, D., Pizzo, V.J., Arge, C.N.: 2004, J. Geophys. Res. 110, A2. doi:10.1029/2004JA010745.

Owens, M.J.: 2006, J. Geophys. Res. 111, A12109.

Pevtsov, A.A.: 2002, In: Martens, P.C.H., Cauffman, D. (eds.) Multi-Wavelength Observations of Coronal Structure and Dynamics, COSPAR Colloquia Series 13, 125.

Rust, D.M.: 2004, Geophys. Res. Lett. 21, 241.

Richardson, I.G., Cane, H.V.: 2010, Solar Phys. 264, 189.

Qiu, J., Hu, Q., Howard, T.A., Yurchyshyn, V.B.: 2007, Astrophys. J. 659, 758.

Schrijver, C.: 2007, Astrophys. J. Lett. 655, 117.

Tang, F.: 1987, Solar Phys. 107, 233.

Thernisien, A., Howard, R.A., Vourlidas, A.: 2006, Astrophys. J. 652(1), 763 - 773.

Vandas, M., Fischer, S., Geranios, A.: 1991, Planet. Space Sci. 39, 1147.

Webb, D.F., Cliver, E.W., Crooker, N.U., St. Cyr, O.C., Thompson, B.J.: 2000, J. Geophys. Res. $105,7491$.

Welsch, B.T., Li, Y.: 2008, In: Howe, R., Komm, R.W., Balasubramaniam, K.S., Petrie, G.J.D. (eds.) Subsurface and Atmospheric Influences on Solar Activity, ASP Conf. Ser. 383, 429.

Welsch, B.T., Li, Y., Schuck, P.W., Fisher, G.H.: 2009, Astrophys. J. 705, 821.

Yurchyshyn, V., Abramenko, V., Tripathi, D.: 2009, Astrophys. J. 705, 426.

Yurchyshyn, V., Hu, Q., Lepping, R.P., Lynch, B.J., Krall, J.: 2007, Adv. Space Res. 40, 1821.

Zhang, G., Burlaga, L.F.: 1988, J. Geophys. Res. 93, 2511.

Zhao, X., Hoeksema, J.T.: 1996, J. Geophys. Res. 101, 4825. 